\title{
Effects of roasting on phenolics composition and antioxidant activity of peanut (Arachis hypogaea $L$.) kernel flour.
}

\begin{abstract}
The effects of roasting on the phenolics composition and antioxidant activity of peanut (Arachis hypogaea L.) kernel flour were appraised. Peanut kernel flour, with and without skin, were roasted at $160{ }^{\circ} \mathrm{C}$ for $10,20,30,40$ and $50 \mathrm{~min}$. The resultant changes in the antioxidant activity of roasted peanut kernel flour were assessed by the determinations of total phenolics, 1,1-diphenyl-2-picrylhydrazyl free radical-scavenging capacity, percent inhibition of linoleic acid oxidation and thiobarbituric acid test and compared with those of unroasted kernel flour. It was observed that roasting significantly $(p<0.05)$ increased the antioxidant activity of the peanut kernel flour. HPLC analysis revealed the detection of three phenolic acids ( $\mathrm{p}$ hydroxybenzoic, chlorogenic, p-coumaric), two flavonols (quercetin, kaempferol), and a stilbene (resveratrol) both in the roasted and unroasted samples. In peanut kernel flour without skin, the contents of the phenolics increased in the initial roasting phase, however, decreased gradually in the later phase (>20 min of roasting time). In contrast, over the course of heating, the amounts of phenolics were noted to be slightly increased in the peanut kernel flour with skin; the most significant $(\mathrm{p}<0.05)$ increase occurred in the concentration of $\mathrm{p}$ coumaric acid and quercetin at 30,40, and 50 min of roasting. The results of this study reveal that optimum roasting time should be sought to enhancing the antioxidant capacity and phenolics concentration in peanut kernel flour.
\end{abstract}

Keyword: Peanut kernel; Roasting time; TPC; Colorimetric antioxidant assays; Phenolics acids; Flavonols. 